Ann. Zootech., 1986, 35 (4), 329-340

\title{
Distribution nycthémérale des vêlages : résultats d'une enquête auprès des éleveurs
}

\author{
M.J. BOSC, Y. VASLET de FONTAUBERT \\ I.N.R.A., Station de Physiologie de la Reproduction, 37380 Monnaie, France
}

\begin{abstract}
Résumé
La distribution nycthémérale de 1574 vêlages a été étudiée à la suite d'une enquête réalisée dans 21 élevages au cours de deux années successives. Dans la majorité des élevages, les vêlages suivent une distribution horaire uniforme. Le découpage de la journée en 4 périodes de 6 heures confirme ce résultat; il indique une variation annuelle moyenne de $8 \mathrm{p} .100$ par période et cette dernière peut néanmoins atteindre 20 p. 100 inter ou intra élevages. La fréquence moyenne des vêlages diurnes (de $6 \mathrm{~h}$ à $20 \mathrm{~h}$ ) correspond aussi à celle qui est déduite d'une répartition uniforme. La variabilité observée est cependant non négligeable car elle peut atteindre $20 \mathrm{p} .100$ entre élevages et 30 p. 100 entre les deux années concernées. Dans deux élevages, les enregistrements effectués pendant plusieurs années ont permis d'apprécier la répétabilité individuelle de l'heure du vêlage. L'analyse indique que 4 vêlages successifs se répartissent au hasard au cours du nycthémère.
\end{abstract}

Mots clés : Vêlage, distribution, nycthémère, heure, répétabilité.

\section{Introduction}

Assurer dans de bonnes conditions le déroulement du travail et le cas échéant, l'intervention du clinicien, s'assurer de l'ingestion du colostrum par le nouveau-né, lui donner les premiers soins, constituent l'essentiel du travail de l'éleveur lors d'une mise bas. Ces nécessités contraignent l'éleveur à une surveillance qui dépend de la variabilité de la durée de la gestation et de celle du moment de la naissance au cours du nycthémère. Chez les bovins domestiques, la plupart des études récentes (EDwards, 1979 ; Bosc, 1980 ; YaRneY et al., 1982 ; OWENs et al., 1984, 1985 ; McMillan et al., 1985) fait état d'une distribution uniforme des vêlages au cours de la journée. Ces études ont été réalisées dans des élevages expérimentaux et elles ne permettent pas d'apprécier la variabilité qui peut exister, dans le monde professionnel, entre élevages et entre deux années consécutives dans un même élevage. Pour ces raisons, nous avons réalisé une enquête dans 25 élevages; nous rapportons ici les résultats obtenus. Nous avons par ailleurs apprécié si les vêlages successifs d'un animal se sont produits aux mêmes moments de la journée ou s'ils se sont répartis au hasard au cours du nycthémère. Cette analyse de répétabilité a pu être faite grâce aux enregistrements réalisés dans deux des élevages pendant plusieurs années. 


\section{Matériel et méthodes}

L'enquête a été réalisée au cours de deux années consécutives. Elle a consisté en un relevé de la date du vêlage, de l'heure à laquelle il s'est déroulé ou des heures entre lesquelles il a été constaté. Ont aussi été notés, le sexe et l'état du veau à la naissance, l'expulsion ou la rétention du placenta et les conditions de déroulement de la mise bas.

\section{A. Conditions de l'enquête}

Cette enquête a été réalisée chez 25 éleveurs volontaires. A la suite du recueil des dossiers, 4 élevages ont été écartés de l'analyse à cause du taux trop élevé des heures de vêlage inconnues et à cause de l'absence d'indications précises des périodes de la journée auxquelles il aurait pu se produire. L'analyse a donc porté sur 21 élevages ; 10 (groupe I) sont situés dans le Nord-Est de la France (Département des Ardennes essentiellement) et 11 (groupe II) dans le Centre Ouest (Départements de la Mayenne et de la Sarthe).

Les troupeaux du groupe I ont un effectif moyen de 55 animaux $( \pm 15)$. Ceux-ci, de race Charolaise, sont utilisés pour la production de viande. Ils sont maintenus en stabulation (libre et/ou entravée selon l'élevage) du mois de novembre jusqu'au mois de mai, moment auquel ils retournent en herbage. Dans le groupe II, l'effectif moyen des troupeaux est de 20 animaux $( \pm 8)$. Ceux-ci, de race Maine-Anjou, sont exploités pour la production de viande ( 2 élevages) ou de lait ( 3 élevages) ou pour les deux (6 élevages). Ils ne sont en stabulation permanente (libre et/ou entravée) que pendant la période hivernale. Les animaux en stabulation des deux groupes et ceux qui sont soumis à la traite dans le groupe II sont alimentés deux fois par jour, excepté dans un élevage du groupe I où les aliments sont distribués en une seule fois, le matin.

Les animaux des deux groupes ont été inséminés pour la plupart lors d'un œstrus naturel. La majorité des mise bas a eu lieu en stabulation. Dans le groupe I, les vêlages sont observés en période hivernale, alors qu'ils sont répartis tout au long de l'année dans le groupe II. Ainsi 90 p. 100 des animaux du groupe I et 48 p. 100 de ceux du groupe II ont vêlé entre les mois de décembre et de mai. La surveillance des préparturientes a été effectuée par rondes fréquentes de jour, moins nombreuses de nuit mais dans la plupart des cas de façon régulière; elle a été réalisée par tous les membres du personnel de l'élevage. Les conditions de vêlage ne sont pas homogènes et les animaux prêts à vêler peuvent être selon le cas et selon l'élevage isolés de leurs congénères. Les caractéristiques des vêlages relevés au cours de l'enquête sont résumées dans le tableau 1. Les taux de veaux vivants à la naissance, ceux de rétentions placentaires et ceux de facilité de vêlage sont peu différents d'un groupe à l'autre.

\section{B. Analyses}

Les analyses ont porté sur les distributions nycthémérales des vêlages. La répartition horaire a été étudiée sur 17 élevages (6 du groupe I et 11 du groupe II) pour lesquels le taux d'heures de mise bas connues avec précision a été supérieur à $90 \mathrm{p}$. 100. Les vêlages qui se sont produits à une heure inconnue n'ont pas été pris en compte dans cette analyse, même si la période au cours de laquelle ils se sont passés a 
TABLEAU 1

Quelques caractéristiques des vêlages relevées au cours de l'enquête.

Calving characteristics recorded during the study.

\begin{tabular}{|c|c|c|c|c|c|c|}
\hline \multirow{2}{*}{$\begin{array}{l}\text { Vêlages } \\
\text { Calvings }\end{array}$} & \multicolumn{3}{|c|}{$\begin{array}{l}\text { Groupe I (10 élevages) } \\
\text { Group I (10 herds) }\end{array}$} & \multicolumn{3}{|c|}{$\begin{array}{l}\text { Groupe II (11 élevages) } \\
\text { Group II (1I herds })\end{array}$} \\
\hline & $\begin{array}{l}1 \text { veau } \\
1 \text { calf }\end{array}$ & $\begin{array}{l}2 \text { veaux } \\
2 \text { calves }\end{array}$ & $\begin{array}{l}\text { Total } \\
\text { Total }\end{array}$ & $\begin{array}{l}1 \text { veau } \\
1 \text { calf }\end{array}$ & $\begin{array}{l}2 \text { veaux } \\
2 \text { calves }\end{array}$ & $\begin{array}{l}\text { Total } \\
\text { Total }\end{array}$ \\
\hline $\begin{array}{l}\text { Nombre } \ldots \ldots \ldots \ldots \ldots \ldots \\
\text { Number }\end{array}$ & 1057 & 58 & 1115 & 415 & 44 & 459 \\
\hline $\begin{array}{l}\% \text { veaux vivants } \ldots \ldots \ldots \ldots \\
\% \text { of alive calves }\end{array}$ & 96,8 & 90,5 & 96,2 & 94,7 & 81,8 & 93,5 \\
\hline $\begin{array}{l}\% \text { rétentions placentaires } \ldots \ldots \\
\% \text { placenta retention }\end{array}$ & 2,6 & 34,5 & 3,4 & 4,1 & 36,4 & 5,5 \\
\hline $\begin{array}{l}\% \text { vêlages faciles } \ldots \ldots \ldots \ldots \\
\% \text { easy calvings }\end{array}$ & 96,7 & 100,0 & 96,9 & 95,2 & 90,9 & 95,0 \\
\hline $\begin{array}{l}\% \text { vêlages difficiles } \ldots \ldots \ldots \ldots \\
\% \text { difficult calvings }\end{array}$ & 2,8 & - & 2,7 & 4,6 & - & 4,3 \\
\hline $\begin{array}{l}\% \text { césariennes } \ldots \ldots \ldots \ldots \ldots \\
\% \text { caesarian sections }\end{array}$ & 0,5 & - & 0,4 & 0,2 & 9,1 & 0,7 \\
\hline
\end{tabular}

été enregistrée. Le test de Kolmogorov-Smirnov (SIEGEL, 1956) a alors été utilisé pour comparer les distributions observées entre elles ou avec une distribution théorique uniforme (soit une probabilité horaire de vêlage égale à $p_{i}=1 / 24$ ). L'analyse a ensuite été effectuée sur ces mêmes élevages après découpage des 24 heures en 4 périodes égales de 6 heures ou en 2 périodes inégales dont l'une de $14 \mathrm{~h}$ (entre $6 \mathrm{~h}$ et $20 \mathrm{~h}$ ou jour) et l'autre de $10 \mathrm{~h}$ (entre $20 \mathrm{~h}$ et $6 \mathrm{~h}$ ou nuit); dans ce dernier cas tous les élevages ont été considérés. Le test de $X^{2}$ (Vessereau, 1960) a alors été employé pour comparer les élevages entre eux et avec une distribution théorique uniforme $\left(p_{i}=1 /\right.$ 24). Compte tenu des effectifs par élevage et de la répartition mensuelle des vêlages, la comparaison entre les deux années d'observation n'a été faite que sur les troupeaux du groupe I.

\section{Analyse de la répétabilité de l'heure du vêlage}

Nous avons pu faire cette analyse de répétabilité grâce aux données recueillies pendant plusieurs années par deux éleveurs du groupe I ; nous avons alors retenu les animaux ayant eu 4 vêlages successifs. Pour chaque animal, chaque heure de vêlage a été définie selon ses coordonnées polaires selon l'exemple de la figure 1a, les heures de vêlage étant distribuées sur une circonférence de centre 0 et de rayon 1 . Après avoir 


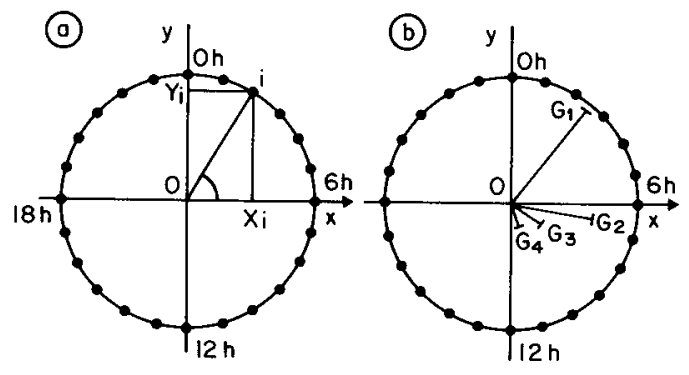

FIG. 1

Estimation de la répétabilité de l'heure du vêlage pour un animal.

Utilisation des coordonnées polaires (Ia) de l'heure du vêlage et calcul du barycentre de 4 vêlages successifs. Présentation (1b) de 4 exemples de calcul.

Estimation of calving time repeatability in an animal using polar coordinates of calving hour (1a) and determination of the barycenter of 4 successive calvings.

Presentation (1b) of 4 examples of calculations.

affecté chaque heure d'un coefficient de masse égal à 1 , les coordonnées du barycentre Gj d'un animal (j) ont été calculées avec

$$
\begin{aligned}
& x j=\sum_{i} \cos \theta i / n i \text { avec } 1 \leqslant i \leqslant 4 \\
& y_{j}=\sum_{i} \sin \theta i / n i
\end{aligned}
$$

et

$$
\begin{aligned}
& 1 \overline{0 G}_{j} 1=\sqrt{x^{2}+y^{2}} \\
& \theta_{j}=\operatorname{arctg} \frac{y}{x}
\end{aligned}
$$

Avec ce procédé, on peut déduire aisément qu'un animal (m) vêlant 4 fois consécutives à la même heure $(m)$ définit un barycentre $G m$ tel que $O G m=1$ et un angle $\theta \mathrm{m}$. De même, un animal (n) vêlant 4 fois consécutives à 4 moments répartis sur les sommets d'un carré définit un barycentre $\mathrm{Gn}$ avec $\mathrm{OGn}=0$ et un angle $\theta \mathrm{n}=0$.

La figure 1b présente 4 barycentres G1 - G2 - G3 - G4 illustrant les 4 répartitions horaires suivantes :

$$
\begin{aligned}
& 1^{\mathrm{er}} \text { cas }: 1,2,3 \text { et } 4 \mathrm{~h}(\mathrm{G} 1) \\
& 2^{\mathrm{e}} \text { cas }: 2,5,8 \text { et } 11 \mathrm{~h}(\mathrm{G} 2) \\
& 3^{\mathrm{e}} \text { cas }: 2,6,11 \text { et } 16 \mathrm{~h}(\mathrm{G} 3) \\
& 4^{\mathrm{e}} \text { cas }: 3,7,17 \text { et } 20 \mathrm{~h}(\mathrm{G} 4)
\end{aligned}
$$

Ces exemples montrent que les coordonnées du barycentre sont représentatives de la variabilité des heures de vêlage. Pour chaque élevage, nous avons ensuite calculé la moyenne des coordonnées barycentriques avec

$$
\begin{aligned}
& \theta=\sum_{\mathrm{j}} \theta_{\mathrm{j}} / \mathrm{m} ; 1 \leqslant \mathrm{j} \leqslant \mathrm{m} \text { (effectif retenu) } \\
& \overline{10 \mathrm{G}} 1=\sum_{\mathrm{j}} \overline{10 \mathrm{G}_{\mathrm{j}}} 1 / \mathrm{m} ; 1 \leqslant \mathrm{j} \leqslant \mathrm{m} \text { (effectif retenu) }
\end{aligned}
$$


Nous avons alors comparé les élevages, séparément ou en groupe, aux résultats obtenus après tirage au sort de 4 valeurs représentant 4 vêlages successifs (analyse de variance, VESSEREAU, 1960).

\section{Résultats}

L'analyse des distributions horaires des vêlages indique qu'un seul élevage appartenant au groupe II s'écarte significativement $(\mathrm{p}<0,05)$ d'une distribution uniforme, lorsque les deux années d'observation sont considérées en pool ; cet élevage présente l'effectif le plus faible. Dans chaque groupe, les élevages ne diffèrent pas entre eux $(p>0,05)$ excepté pour deux d'entre eux du groupe II $(p<0,05)$. Un seul élevage du groupe I présente une variation significative entre les 2 années d'observation (p< 0,05 ; fig. 2).

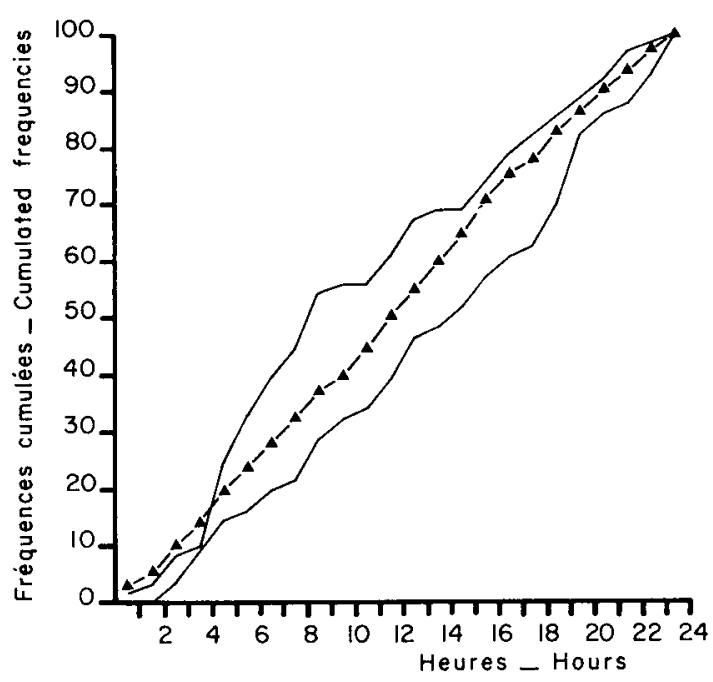

FIG. 2

Répartition nycthémérale des vêlages dans l'élevage du groupe I présentant une variation significative au cours des 2 années d'enquête (Effectifs 56 et 61 animaux). Courbe $(\mathbf{\Delta}-\mathbf{\Delta})$ moyenne obtenue à partir des 6 élevages du groupe I retenus pour l'analyse des distributions horaires.

Circadian distribution of calving times in the herd of group I showing a significant difference

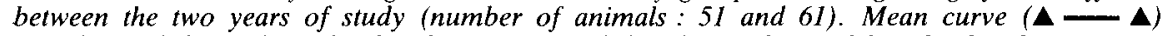
obtained from the 6 herds of group I used for the analysis of hourly distributions.

Après découpage des 24 heures en 4 classes de $6 \mathrm{~h}$ (la $1^{\text {te }}$ débutant à minuit), l'analyse confirme que les 17 élevages précédemment retenus ne diffèrent pas de la distribution uniforme $(p>0,05)$ lorsque les deux années d'observations sont prises en 


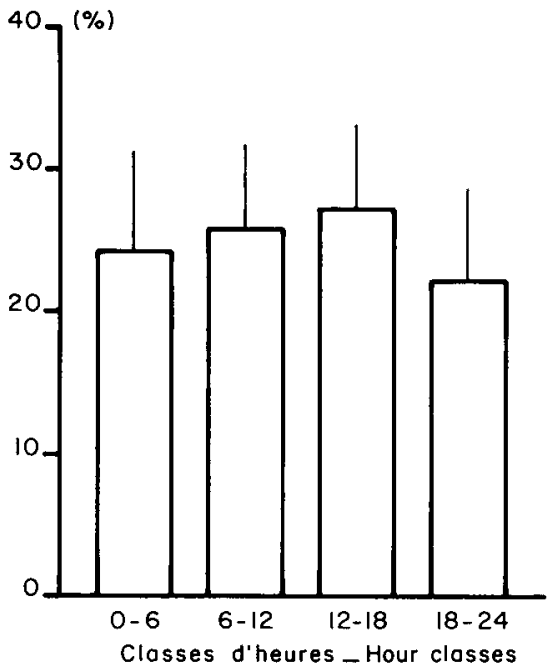

Fig. 3

Répartition (\%) des vêlages de 6 élevages du groupe $I(m \pm s d)$ par classes de 6 heures. Pool des 2 années d'observation.

Distribution (\%) of calvings in 6 herds of group $I(m \pm s d)$ by sets of 6 hours.

Pooled values of two years of study.

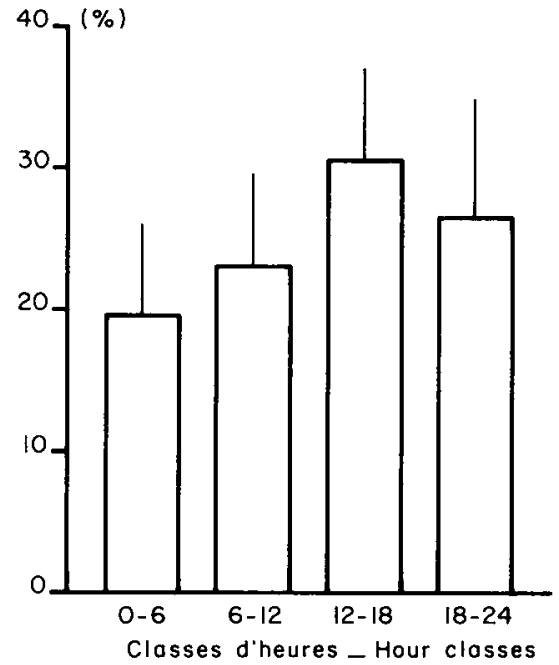

FIG. 4

Répartition (\%) des vêlages des 11 élevages du groupe $I I(m \pm s d)$ par classes de 6 heures. Pool des 2 années d'observation.

Distribution (\%) of calvings in 11 herds of group $I I(m \pm s d)$ by sets of 6 hours. Pooled values of two years of study. 
compte. Les taux moyens de vêlage par classe sont voisins dans les deux groupes et ils sont les plus élevés entre $12 \mathrm{~h}$ et $18 \mathrm{~h}$ (fig. 3 et 4 ). Le taux de vaches vêlant par classe varie d'une année sur l'autre selon une amplitude moyenne de $8 \mathrm{p}$. 100. Les variations intraclasses sont cependant importantes car le coefficient de variation est compris entre 0,53 et 0,93 . Ainsi une variation annuelle atteint à l'extrême 20 p. 100 pour un élevage et une des quatre périodes de la journée.

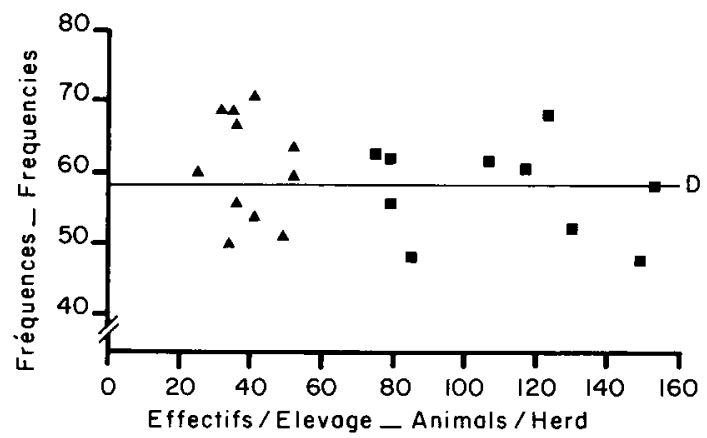

FIG. 5

Fréquence des vêlages diurnes (observés entre $6 h$ et 20 h) dans les 21 élevages. Pool des 2 années d'observation et effectifs totaux.

Frequency of diurnal calvings between 06.00 and $20.00 \mathrm{~h}$ in the 21 herds. Pooled values of the two years of study and numbers of animals per herd.

(A) Elevages du groupe I / Herds of group I. (ロ) Elevages du groupe II / Herds of group II. (D) Fréquence correspondant à la distribution horaire uniforme. Frequency corresponding to the even hourly distribution.

L'analyse des répartitions des mise bas entre le jour $(6 \mathrm{~h}$ à $20 \mathrm{~h})$ et la nuit $(20 \mathrm{~h}$ à $6 \mathrm{~h}$ ) a été faite sur tous les élevages et les résultats sont illustrés dans la figure 5 , pour les deux années d'observation. Dans le groupe I, le taux moyen des vêlages diurnes est égal à 57,7 p. $100( \pm 6,7$ p. 100$)$; il est proche de celui d'une répartition horaire uniforme $(58,3$ p. 100$)$ mais les deux élevages du groupe qui représentent les valeurs extrêmes $(47,7$ et 68,3 p. 100$)$ s'en écartent cependant de façon significative $(p<0,05$ ou $\mathrm{p}<0,01)$. Dans ce groupe, les valeurs extrêmes constatées chaque année d'observation sont du même ordre de grandeur $\left(46,6\right.$ et $76,2 \mathrm{p}$. 100 pour la $1^{\text {re }}$ année et 45,9 et 70,4 p. 100 pour la $2^{c}$ ) mais dans 2 élevages des dix de ce groupe, les taux des vêlages diurnes ou nocturnes ont varié de façon significative entre la $1^{\text {re }}$ et la $2^{\mathrm{e}}$ année $(\mathrm{p}<0,05)$. Dans le groupe II, le taux moyen des vêlages diurnes est égal à $60,7 \mathrm{p} .100$ ( $\pm 7,5$ p. 100); les élevages ne diffèrent pas entre eux ni de la répartition horaire uniforme $(p>0,05)$. Les taux extrêmes constatés entre les 11 élevages de ce groupe (II) sont voisins de ceux qui ont été observés dans le groupe I $(50,0$ et 70,7 p. 100).

L'étude de la répétabilité de l'heure du vêlage a été effectuée dans deux élevages du groupe I qui ont présenté une répartition uniforme des vêlages au cours du nycthémère. Les valeurs moyennes des coordonnées barycentriques des cas étudiés ne sont pas différentes entre élevages $(p>0,05)$ ni entre les élevages et les valeurs obtenues après tirage au sort $(\mathrm{p}>0,05)$ (tabl. 2). De plus, les distributions des valeurs du module du barycentre des vêlages successifs et celles obtenues après tirage au sort sont équivalentes $(\mathrm{p}>0,05)$ (tabl. 3). 


\section{TABLEAU 2}

Coordonnées polaires moyennes (argument: $\theta$ et module : $\overline{O G} 1$ ) des barycentres représentant 4 vêlages successifs d'un animal dans 2 élevages du groupe $I$. Comparaison avec les résultats obtenus par tirage au sort.

Mean polar coordinates of barycenters representative of 4 successive calvings of one animal. Data obtained in two herds of group I. Comparison with results obtained after random drawing.

\begin{tabular}{|c|c|c|c|c|c|c|}
\hline \multirow[b]{3}{*}{$\begin{array}{l}\text { Nombre d'individus } \\
\text { Number of animals }\end{array}$} & \multicolumn{3}{|c|}{$\begin{array}{l}\text { Elevages } \\
\text { Herds }\end{array}$} & \multicolumn{3}{|c|}{$\begin{array}{l}\text { Tirages au sort } \\
\text { Random drawings }\end{array}$} \\
\hline & A & B & $A+B$ & A & B & $A+B$ \\
\hline & 21 & 18 & 39 & 21 & 18 & 39 \\
\hline $\begin{array}{l}\text { Argument } \theta \\
\text { Argument }\end{array}$ & $-2,0$ & 2,9 & 0,3 & $-8,6$ & $-4,5$ & $-6,7$ \\
\hline $\begin{array}{l}\text { Module } \overline{\mathrm{OG}} 1 \ldots \ldots \ldots \ldots \\
\text { Modulus }\end{array}$ & 0,52 & 0,40 & 0,47 & 0,45 & 0,48 & 0,46 \\
\hline
\end{tabular}

TABLEAU 3

Distribution (\%) des modules du vecteur définissant le barycentre de 4 vêlages successifs pour un animal, dans 2 élevages du groupe I. Comparaison avec un tirage au sort.

Distribution (\%) of the vector modulus defining the barycenter of 4 successive calvings of one animal in two herds of group I. Comparison with a random drawing.

\begin{tabular}{l|c|c|c|c|c}
\hline \hline Valeurs de (Values of) $1 \overline{0 \mathrm{G}} 1$ & 0,00 à 0,19 & 0,20 à 0,39 & 0,40 à 0,59 & 0,60 à 0,79 & 0,80 à 1,0 \\
\hline $\begin{array}{l}\text { Elevages }(\mathrm{A}+\mathrm{B})(39 \text { cas }) \\
\text { Herds }(A+B)(39 \text { cases) }\end{array}$ & 17,9 & 20,5 & 30,8 & 20,5 & 10,3 \\
$\begin{array}{l}\text { Tirage au sort }(39 \text { cas }) \ldots \\
\text { Random drawing (39 cases) }\end{array}$ & 12,8 & 30,8 & 30,8 & 12,8 & 12,8 \\
\hline \hline
\end{tabular}

\section{Discussion - Conclusion}

Dans cette étude, la plupart des élevages présente une répartition nycthémérale des vêlages équilibrée et proche d'une distribution horaire uniforme. Les écarts significatifs à cette dernière sont limités à quelques cas dont un est imputable au faible effectif de l'élevage. Les variations annuelles des fréquences de mise bas par périodes de 6 heures sont en moyenne de l'ordre de 8 p. 100 ; cependant, elles peuvent atteindre 20 p. 100 
dans un même élevage ou entre deux élevages. L'analyse de la répétabilité de l'heure du vêlage indique que les vêlages successifs d'un même animal se répartissent d'une manière aléatoire au cours du nycthémère. Cette absence apparente de répétabilité conforte les observations de distribution uniforme constatées dans les deux élevages concernés par cette analyse, comme dans la plupart des autres élevages soumis à l'enquête.

L'enquête fait ressortir une très légère tendance favorisant les vêlages diurnes. En effet, les vêlages ont été plus fréquents entre $6 \mathrm{~h}$ et $18 \mathrm{~h}$ qu'entre $18 \mathrm{~h}$ et $6 \mathrm{~h}$ (fig. 3 et 4) et dans 12 élevages sur 21 , la fréquence des vêlages diurnes a été supérieure à la fréquence déduite d'une distribution uniforme (fig. 5). Cette tendance ressort aussi des observations précédentes dans lesquelles elle est plus (Pennington et Albright, 1985) ou moins (McMillan et al., 1985; Yarney et al., 1982) marquée. Cette enquête fait néanmoins ressortir, à propos des vêlages diurnes ou nocturnes, des différences de fréquences non négligeables qui peuvent atteindre 20 p. 100 environ entre élevages et même 30 p. 100 d'une année sur l'autre pour un des élevages.

La distribution uniforme des vêlages constatée dans cette enquête comme dans les études antérieures (EDWARds, 1979 ; Bosc, 1981 ; YARNEY et al., 1982 ; Bosc et al., 1984 ; OWEns et al., 1984/1985 ; McMillan et al., 1985) pose le problème des facteurs susceptibles de la modifier significativement et des moyens à mettre en œuvre pour y parvenir. Cette enquête fait ressortir une variabilité non négligeable mais elle ne permet pas d'en dégager les raisons. Les activités imposées au troupeau liées à la traite (Edwards, 1979) ou à la présence des vachers (Le Neindre et Maronne, 1983) ont été reconnues comme pouvant affecter significativement l'heure du vêlage; le rythme de l'élevage plutôt que le stress de la préparturiente en a été tenu comme responsable.

Par ailleurs, les tentatives de modifications des répartitions diurnes et nocturnes des vêlages par le changement des heures d'alimentation ont été plus ou moins fructueuses selon les études (Lowman et al., 1981 ; Anderson, 1983 ; ClaRK et al., 1983 ; Bosc et $a l ., 1984$ ). Les études les plus récentes à ce sujet (McMillan et al., 1985 ; Pennington et Albright, 1985) ont indiqué une inefficacité apparente des rythmes de distribution alimentaire précédemment proposés. Ces résultats contradictoires suggèrent qu'il est difficile de modifier les rythmes endogènes liés à la routine de l'élevage et que le rythme alimentaire n'est pas en fin de gestation un entraîneur biologique aussi efficace chez la vache que chez la rate préparturiente (Bosc et Nicolle, 1985).

Cette étude souligne à nouveau la différence qui existe entre les bovins domestiques et d'autres espèces de mammifères, à propos des distributions nycthémérales des naissances. A cet égard, les ovins domestiques sont peu différents des bovins (LINDAHL, 1964 ; Bosc, 1980). Certaines espèces sont essentiellement diurnes comme l'alpaga (Bustinza et al., 1970 ; Sumar, 1985) la rate (Bosc, 1981) ou la chèvre (Lickliter, 1984/1985 ; Bosc et al., en préparation) ; d'autres sont essentiellement nocturnes comme la lapine (NaAktgeboren, 1963), la souris (Svorad et Sachova, 1959 ; Porter, 1972) et la jument (Rossdale et ShORT, 1967 ; CAMPITElli et al., 1982/1983). Enfin, il $\mathrm{y}$ a aussi des espèces qui ont des périodes préférentielles d'accouchement sans être essentiellement diurnes ou nocturnes : ce sont les cas de l'espèce humaine ou des porcins domestiques (Bosc, 1980). Tous ces exemples de rythme nycthéméral des naissances suggèrent que les conditions de milieu perçues par la mère influencent les mécanismes d'initiation de la naissance et/ou ceux qui contrôlent le déroulement du travail et par conséquent sa durée. Le travail a une durée encore mal appréciée chez les mammifères domestiques (Bosc, 1984) et on ignore si elle peut varier de façon à 
déterminer un rythme nycthéméral caractéristique des naissances. Quant au mécanisme d'initiation de la naissance, il implique chez la vache comme chez la brebis et la chèvre, un mécanisme endocrinien fœtal dont la production de cortisol est un maillon essentiel (Bosc, 1984). L'indépendance relative du compartiment foetal et de son système hypothalamo-hypophyso-surrénalien pourrait alors être une des raisons des distributions nycthémérales uniformes des naissances constatées chez les bovins.

D'un point de vue concret, la surveillance des vaches préparturientes doit s'exercer aussi bien de jour que de nuit. Pour limiter les contraintes qui lui sont imposées, l'éleveur peut prévoir de façon précise le moment du vêlage ; il peut aussi le reporter en dehors des heures indésirables ou le provoquer avant terme (Bosc, 1984). La modification des fréquences des vêlages nocturnes et diurnes demeure cependant un objectif attrayant. Il importe alors de connaître puis de mettre en cuvre les facteurs d'environnement capables de modifier le moment du vêlage en tenant compte des variations aléatoires auxquelles il est soumis.

Reçu en mai 1986.

Accepté en août 1986.

\title{
Remerciements
}

Les auteurs remercient Mrs J. Ponsart et H. Laurent de l'Etablissement Départemental de l'Elevage des Ardennes (Charleville-Mézières), Mr B. Chretien de l'U.P.R.A. Maine-Anjou (Château-Gonthier) et tous les éleveurs grâce auxquels cette enquête a été réalisée.

\author{
Summary \\ Circadian distribution of calvings : \\ results of a survey in commercial herds
}

The circadian distribution of 1574 calvings was studied from a survey performed in 21 herds during two successive years. In most herds, calvings followed an hourly uniform distribution. This was confirmed when dividing the day into 4 periods of 6 hours. The annual variation averaged 8 p. 100 per period, but it could reach 20 p. 100 between and within herds. The mean frequency of diurnal calvings $(06.00$ to $20.00 \mathrm{~h})$ was similar to that deduced from an even distribution, but the observed variability reached $20 \mathrm{p}$. 100 between herds and $30 \mathrm{p}$. 100 between two years in one herd. Records over several years in two herds allowed to estimate the individual repeatability of calving time. According to the analysis, 4 successive calvings were randomly distributed over the day.

Key words : Calvings, hourly distribution, circadian distribution, repeatability. 


\section{Références bibliographiques}

Anderson V.L., 1983. Effect of feeding time on calving time. North Dakota Farm Research, 41, 28-29.

Bosc M.J., 1980. Influence de la photoperiode ou des conditions de milieu sur la parturition. In : "Rythmes et Reproduction". Rapporteurs généraux R. Ortavant, A. Reinberg, ed. Masson, Paris, 179-193.

Bosc M.J., 1981. Effects of photoperiod on the time of birth in the rat. In : " Photoperiodism and Reproduction ». Les colloques de l'I.N.R.A. $\mathrm{n}^{\circ}$ 6. Ed. R. Ortavant, J.P. Ravault, 33-50, I.N.R.A., Route de Saint-Cyr, Versailles.

Bosc M.J., 1984. Les mécanismes de la naissance chez la brebis, la chèvre, la truie et la vache. Contrôle et maîtrise de la naissance. In "Physiologie et Pathologie périnatales chez les animaux de ferme ». Ed. R. Jarrige, I.N.R.A., Paris, 3-30.

Bosc M.J., Cochaud J., Cornu Cl., Touze J.L., Vaslet de Fontaubert Y., 1984. Control of lambing and calving. In : "The Reproductive potential of cattle and sheep ". Les colloques de l'I.N.R.A. n ${ }^{\circ}$ 27. Ed. R. Ortavant and M. Schindler, 295-326, I.N.R.A., Route de Saint-Cyr, Versailles.

BosC M.J., Nicolle A., 1985. Influence of photoperiod on the time of birth in the rat. IV. Effects of an imposed feeding rhythm. Reprod. Nutr. Develop., 25, 39-48.

Bosc M.J., Bourgy G., Gulluimin P., Pignon P., 1986. La répartition des chevrotages au cours du nycthémère (en préparation).

Bustinza J., Gallegos M., Santos M.A., 1970. Observaciones del parto de Alpaca. Anales de la Primera Convencion Sobre Camedidos Sudamericanos (Auquenidos) Puno-Perou, 153-155.

Campitelu S., Carenzi C., Verga M., 1982/1983. Factors which influence parturition in the mare and development of the foal. Appl. Anim. Ethol., 9, 7-14.

Clark A.K., Spearow A.C., Owens M.J., 1983. Relationship of feeding time to time of parturition for dry holstein cows. J. Dairy Sci., 66, (supplt 1), p. 138 (Abstr. p. 78).

Edwards S.A., 1979. The timing of parturition in dairy cattle. J. Agric. Sci. Camb., 93, 359-363.

Le Neindre P., Maronne P., 1983. Répartition des vêlages au cours du nycthémère. Bull. Tech. C.R.V.Z. Theix I.N.R.A., 51, 5-6.

LiCKLITER R.E., 1984/1985. Behaviour associated with parturition in the domestic goat. Appl. Anim. Behav. Sci., 13, 335-345.

Lindahl I.L., 1964. Time of parturition in ewes. Anim. Behav., 12, 231-234.

Lowman B.G., Hankey M.S., Scott N.A., Deas D.W., Hunter E.A., 1981. Influence of time of feeding on time of parturition in beef cows. Vet. Rec., 109, 557-559.

MacMillan, Pearce M.G., Copeman P.J.A., MacDonald K.A., Kilgour R., 1985. Effects of varying feeding time on diurnal calving patterns in dairy cows. Proc. New Zealand Soc. Anim. Prod., 45, 35-37.

NAaktgeboren C., 1963. Untersuchungen über die geburt der saügetiere. Th. Univ. Amsterdam., $50 \mathrm{p}$.

OWens J.L., Edey T.N., Bindon B.M., PiPer L.R., 1984/1985. Parturient behaviour and calfsurvival in a herd selected for twinning. Appl. Anim. Behav. Sci., 13, 321-333.

Pennington J.A., Albright J.L., 1985. Effect of feeding time, behavior and environmental factors on the time of calving in dairy cattle. J. Dairy Sci., 68, 2746-2750.

Porter D.G., 1972. The light regimen and gestation length in the mouse. J. Reprod. Fert., 28, 9-14.

Rossdale P.D., Short R.V., 1967. The time of foaling of thoroughbred mares. J. Reprod. Fert., 13, 341-343.

SiEgel. S., 1956. In : "Non parametric statistics for the behavioral sciences". Int. Student. Ed. McGraw-Hill Book Comp. Inc. New York, 312 pp.

Sumar J., 1985. Reproductive physiology in South American Camelids. In : "Genetics of Reproduction in sheep». Ed. R.B. Land, D.W. Robinson, Butterworths London, $427 \mathrm{pp}$. 
Svorad D., Sachova V., 1959. Periodicity of the Commencement of birth in mice and the influence of light. Physiol. Bohemoslov., 8 (5), 439-442.

Vessereau A., 1960. In : Recherche et Experimentation en Agriculture. Tome 2, Méthodes statistiques en Biologie et en Agronomie. Ed. J.B. Baillères et Fils, Paris, 539 pp.

Yarney T.A., Rahnefeld G.W., Parker R.J., Palmer W.M., 1982. Hourly distribution of time of parturition in beef cows. Can. J. Anim. Sci., 62, 597-605. 